\title{
CHALLENGES IN INDIAN CURRENCY DENOMINATION RECOGNITION \& AUTHENTICATION
}

\author{
Syed Ejaz Ali ${ }^{1}$, MrigankaGogoi ${ }^{2}$, Subra Mukherjee ${ }^{3}$ \\ ${ }^{1}$ PG student, Dept. of ECE, School of Technology, Assam Don Bosco University, Guwahati, India \\ ${ }^{2}$ Asst. Professor, Dept. of ECE, School of Technology, Assam Don Bosco University, Guwahati, India \\ ${ }^{3}$ Asst. Professor, Dept. of ECE, School of Technology, Assam Don Bosco University, Guwahati, India
}

\begin{abstract}
Automatic currency recognition and authentication has become an impending challenge today particularly because of the prevailing fraudulent activities as it hampers our economy. According to the RBI report 435,607 fake notes has been detected in year 2010-2011 and the number is only increasing with technological advancements in the field of printing. Image processing techniques such as texture based, pattern or color based, character recognition etc using different operator or tools such as Prewitt or Sobel or Canny edge detector, ANN, heuristic analysis, SVM etc are commonly used for recognition and authentication of paper currency note. Despite several researches it still remains an open challenge. This paper intends to present an extensive survey of the recent technological trends in recognition and authentication of paper currency note while identifying the various challenges.
\end{abstract}

Keywords - Currency recognition, Currency Authentication, feature extraction,Currency Authentication and Recognition System (CARS)

\section{INTRODUCTION}

The expansion of modern banking services necessitates the needs for automatic currency recognition and authentication system, thus encouraging many researchers to develop high accuracy, reliable and high processing speed techniques. To detect the authenticity of currency note there are two methods i.e first line inspection method and second line inspection method. First line inspection method includes varied density watermarks, ultraviolet fluorescence, intaglio printing, microtext and hologram while the second line inspection methods include isocheck/isogram, fiber based certificates of authenticity, color and feature analysis. First line inspection method is used for on-spot authentication of notes but is easier to counterfeit than the second line inspection method while the second line inspection method requires an extra device to perform the inspection.

The main challenge for currency recognition is to identify currency note in a bunch of currency notes where there is a probability of finding different denomination note. Also a robust system should be able to recognize worn, blurry, defaced and even damaged note during circulation by human being.

\section{CURRENCY NOTE AUTHENTICATION METHOD}

The Currency note Authentication method can be broadly classified into: 1) First Line Inspection Method

2) Second Line Inspection Method

\subsection{First Line Inspection Method}

The various first line inspection methods are discussed below:

a) Watermarks: The Mahatma Gandhi series of banknotes contain Mahatma Gandhi watermark. The banknote paper has a quality of varying density and is printed in a controlled manner. When bright light is illuminated on the rear side of the note the watermark become visible on the other side of the note due to varying light intensity due to varying density [1].

b) Ultraviolet fluorescence: The security thread, some of the fibers embedded on the note between watermark $\&$ denominational value and the serial number of the note are printed in fluorescence ink which becomes bright when illuminated with UV led from the rear side of the note [1].

c) Intaglio printing: In intaglio printing the note is subjected to high pressure printing process which raises the paper surface. The portrait of Mahatma Gandhi, the Reserve bank seal, guarantee and promise clause, Ashoka pillar emblem, RBI governor signature are printed in intaglio, which can be felt by touch in Rs 20, Rs 50, Rs 100, Rs 500 and Rs 1000 currency notes. A special feature in intaglio known as identification mark has been introduced on left of the watermark window on all notes except Rs 10. This feature is in different shapes for different denomination (Rs 20-Vertical rectangle, Rs 50-Square, Rs 100-Triangle, Rs 500-Circle, Rs 1000-Diamond). It helps visually impaired to identify the denomination by touching [1]. 
d) Micro-lettering: Some texts are printed in less than 1 point size between vertical band and Mahatma Gandhi portrait. It is readable only with magnifying glass. In Rs 5 and Rs 10 note the word 'RBI' is present. In the notes Rs 20 and above it also contain the denominational value in microletters [1].

e) Security thread: The security thread appears to the left of the Mahatma Gandhi portrait. The Rs 5, Rs 10, Rs 20 and Rs 50 notes contains a readable, fully embedded windowed security thread with inscription 'Bharat' (in Hindi) and 'RBI'. The Rs 100, Rs 500 and Rs 1000 notes contains readable, windowed security thread alternatively visible on obverse with inscription 'Bharat'(in Hindi) and 'RBI' but the Rs 1000 note contain additional inscription ' 1000 ' in the note[1].

f) Optically variable ink: A new security feature has been added to notes of Rs 500 and Rs 1000 in November 2000. The numeral 500 and 1000 are printed with optically variable ink viz, a color shifting ink. The color of the numeral is green when note is held flat but changes to green when note is held at an angle.

g) Identification mark: An identification mark has been introduced in the note for the benefit of the visually impaired people. This mark is in intaglio printing \& it is in different geometrical shapes for various denominations as shown in fig 1.1.

h) Latent image: It is a vertical band present on the right side of Mahatma Gandhi's portrait showing the respective denominational value in numeral when the note is held horizontal at the eye level. i) See through Register: The small floral design printed both on the front (hollow) and back (filled up) of the note in the middle of the vertical band next to the Watermark has an accurate back to back registration. The design will appear as one floral design when seen against the light.

Generally first line inspection method is used by people for on spot verification of authenticity of the note being exchanged. It is an inexpensive method since it doesn't uses any device for verification. But the disadvantages of this method are that it is easy to counterfeit because it is as visible to the counterfeiter as to the verifier [1].

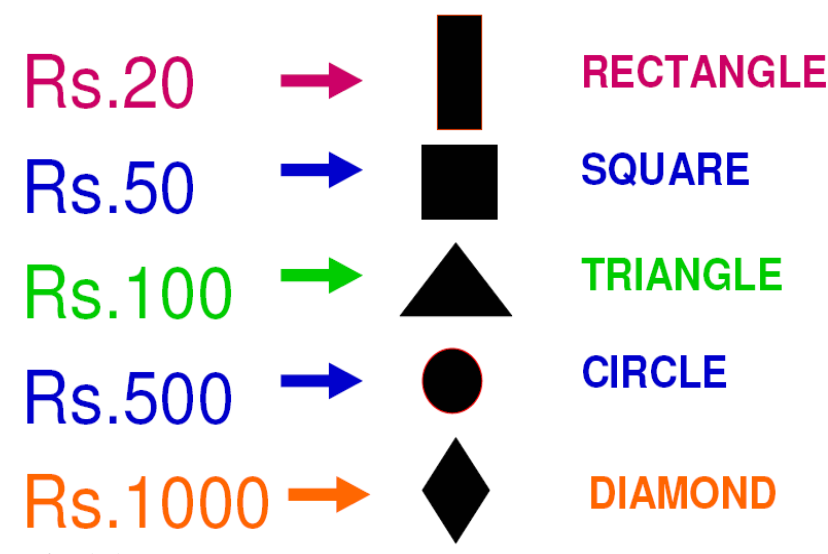

Fig 1.1 Identification mark for various denominations [2]

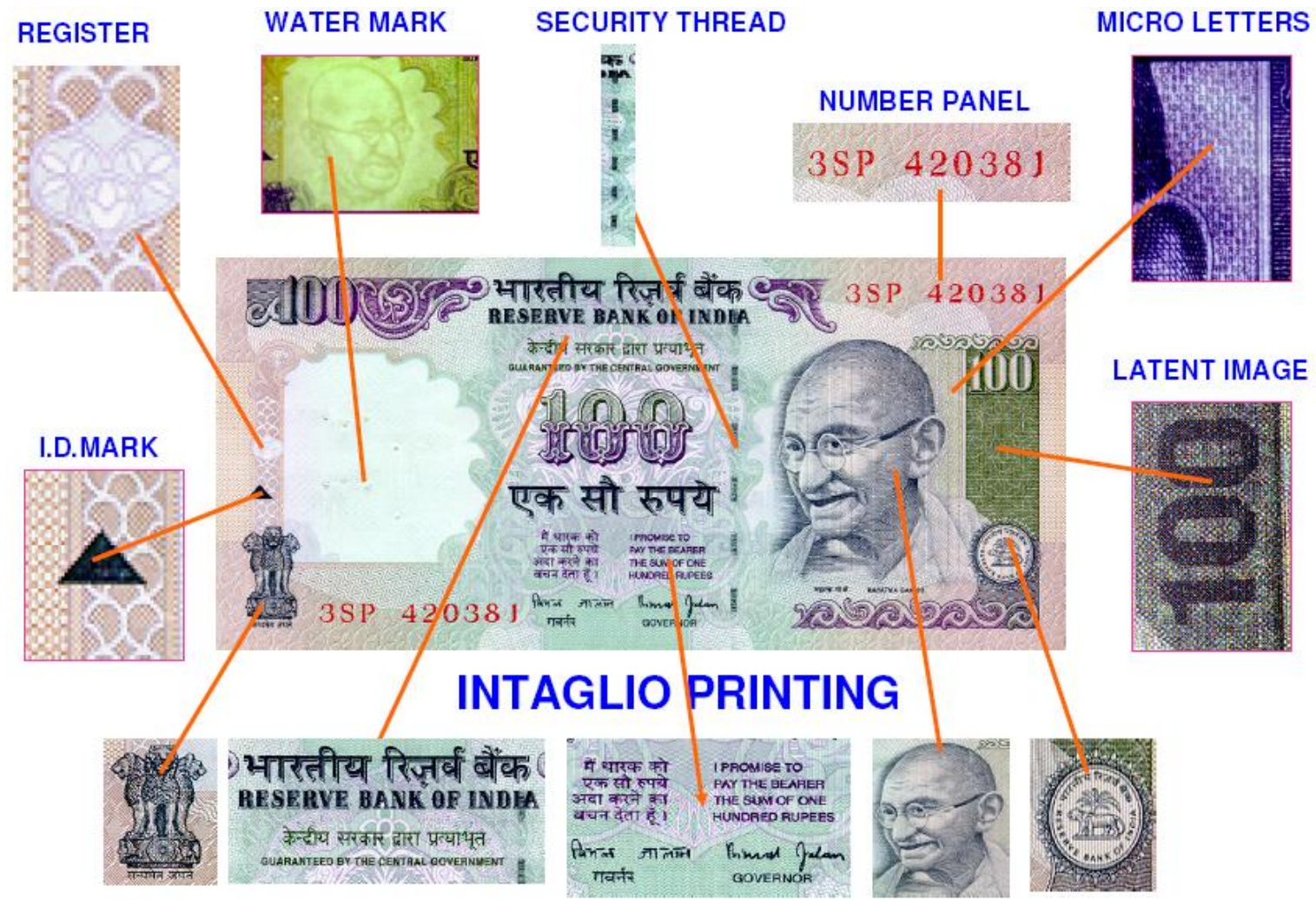

Fig 1.2 Security features in a banknote [2] 

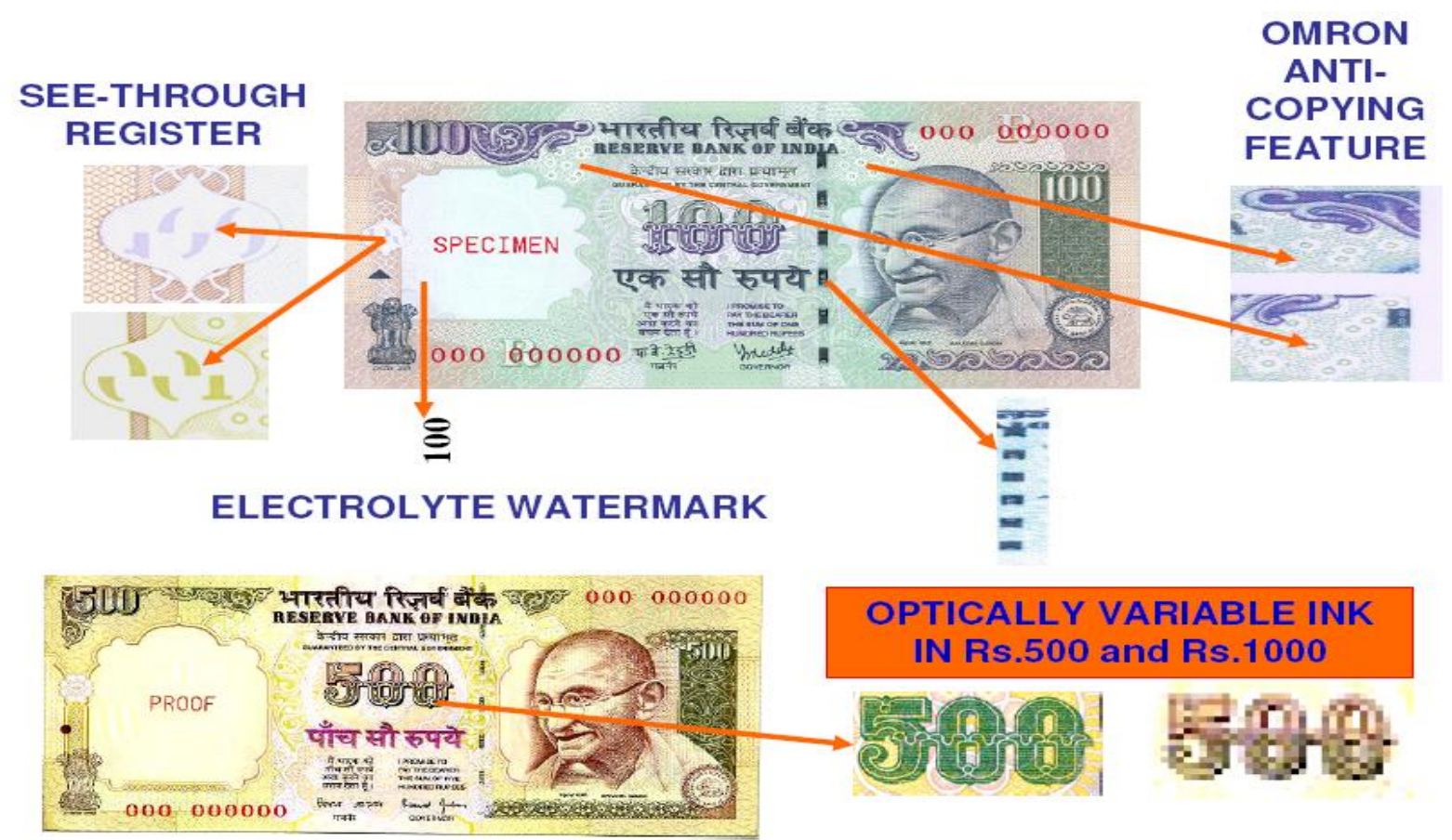

Fig 1.3 New security features added in new M.G series notes[2]

\subsection{Second Line Inspection Method}

a) Counterfeit detection pen: It is a device that is designed to determine whether the note is fake or genuine. The pen contains iodine as ink which is brown in color. The iodine when reacts with starch, which is a primary component that makes white paper looks brighter. The commercial paper is generally brown in color unless it is bleached and starched. The ink will turn black if the note is fake but will remain amber or brown when the note is genuine.

The major limitation of this pen is that when a fake note made from the paper which is not bleached or starched, the pen will detect it as a genuine note [3].

b) Digital image processing: The digital image processing techniques involve extraction of the features from the test image and then comparing with pre stored original image. The different steps in authentication of a note are:

i. Image acquisition: An image is obtained from the digital camera with white background. The main challenge in this image processing will be to process the image with background other than white.

ii. Image preprocessing: The image obtained from the digital camera is too big. In order to reduce the calculation, the size of the image is reduced with the help of image interpolation.

iii. Edge detection: Edge of gray scale image is detected by using any one of the operator such as Sobel, Canny, Prewitt, Roberts and Laplacian of Gaussian methods. Among these operator Canny edge detector is more powerful and can detect true weak edges [4].

iv. Image segmentation: It is a process of dividing the image into its constituent regions or objects. Segmentation algorithm for monochrome images is based on one of the two basic properties of image intensity values i.e Discontinuity and similarity. By using discontinuity property image is partitioned based on abrupt changes in intensity while by using similarity property image is partitioned into regions according to a set of predefined criteria [5].

v. Feature extraction: The main task of feature extraction is to find the correspondingly effective features out of many pending features such as serial no, watermark, security thread, identification mark.

vi. Matching: The extracted features are then compared with original currency features. If the pixel of the segmented image of test currency matches with the segmented image of original currency then the currency is found to be genuine otherwise counterfeit.

In [6], Rubeena Mirza and Vinti Nanda extracted the general attributes of paper currency that is shape including identification mark, security thread and watermark using edge based segmentation method by Sobel operator. After extracting the features the background is separated. The extracted features are compared with extracted features of original test currency by calculating the number of black pixels. 
In another image processing based work [1] recognition of fake note was done by dividing the currency note into some parts and then each part was processed individually using SVM (Support Vector Machines). It is mainly used to determine the location of decision boundaries that produce optimal separation of classes. They also used character recognition to check the serial number of notes with the help of heuristic analysis. Here they have used ANN to train and test the whole system. For advanced authentication they have used UV and infrared light system to detect the watermark, latent image etc.

D. Alekhya, G.Devi Surya Prabha \& G. Venkata Durga Rao has also done fake currency note detection using image processing. Here they first segmented the red, green and blue components of the original and the test currency. Then a new image of combination of different component is taken. But the image with the combination green component of test image is taken since the human eye is sensitive to green. The newly constructed image is compared with the original currency image. Then threshold value of equivalence is calculated to test whether the note is fake or real. When components of two images are combined then if the note tested is original then only at the place of number we get variation otherwise if the tested note is fake then image overlapping will not be proper [3].

In paper [7] and [8] the author used printing technique to authenticate a currency note because different printing processes are used to print different parts of a note. All these printing processes are applied not at a time but in a sequence which is a security aspect. They have used three different classifier i.e. Support Vector Machines (SVM), Neural Network (NN) and Fisher Linear Discrimination Analysis to authenticate the printing process.

In paper [9] the author proposed an effective system to detect counterfeit currency notes by processing the image of currency note taken by IR camera which is illuminated by IR led. By testing 1750 banknotes have attained $95.7 \%$ efficiency.

c) UV illumination method: The currency note has the property that it absorbs the UV light and the fake note reflects the UV light. Therefore this feature can be used for detection of authenticity of currency note. For this a UV transmitter and a receiver or detector is used. The UV light transmitted by the transmitter is absorbed by the genuine note and is reflected by the fake note. Depending upon the light received by the receiver the note is detected as genuine or fake [10].

Another method using UV illumination is a combination of both UV illumination and image processing. Here first the note is illuminated by UV light because some of the patterns are not visible under normal LED illumination. The image obtained is converted into gray image to find the gray histogram. The image is separated into a pattern and a background by using threshold value. There are two methods for separation. Otsu's and Huang Wang's method based on histogram threshold are used in separating an image into two objects. Another method is Gaussian mixture model (GMM) which can segment image into many objects. After this pattern matching is performed to detect the authenticity [10].

The second line inspection methods are more secure and harder to counterfeit than the first line inspection methods which are only visual based. It also adds an extra cost of device to perform verification.

\section{BASIC STEPS OF A GENERAL IMAGE PROCESSING BASED CURRENCY NOTE RECOGNITION METHOD}

The word recognition means to identify objects in an image. The process of recognition starts with image processing techniques. The different steps in currency recognition are:

\section{i. Image acquisition}

It is a process of acquiring image from a currency note by using digital camera. The image is stored for further processing.

\section{ii. Image Pre-Processing}

Image pre-processing is done to enhance some image features important for further processing and analysis. In image pre-processing size of the image is reduced and noise is removed that may have appeared in the image while transferring.

\section{iii. Edge detection}

It aims at identifying points in digital image at which image brightness changes sharply. It is an important step in image processing and analysis.

\section{iv. Binarisation \& Background subtraction}

During binarisation a given image is converted into binary image. Binarisation uses the concept of thresholding where individual pixels in an image is marked as 'object pixels' if their value is greater than the threshold value and as background pixels otherwise. Thus binarisation process leads to background subtraction.

\section{v. Feature extraction}

Feature extraction involves extraction and selection of some effective and important features out of many features which are extremely important in recognition of notes. Some features of an image are:

\section{- Size or Area}

There is a difference in size of different denomination currency note. Therefore size can be used as a feature for currency recognition. But the major limitation of this feature is that size of the image vary depending on the distance from which photo of the image has been taken. To overcome this problem a new feature has been introduced i.e aspect ratio [11]. 


\section{- Aspect Ratio:}

It is the ratio of width of the note to that of height of the note. Unlike the size of the image the aspect ratio of note of particular denomination is independent of the distance from which the photo of the image has been taken.

\section{- Color}

There are varieties of colors present in a currency note. But there is one or two dominant color present in each note which can be used for distinguishing between different currencies note.

\section{- Euler Number}

Euler number is the number of objects in a image minus the total number of holes in an image. MATLAB has in built function to find the Euler number of image [12]

- Texture

There is not much difference in the size and color of different denomination currency of a particular country. So these features may not be able to easily differentiate between different currencies. The Markov chain concept is used to model the texture as random process.

\section{- Matching algorithm}

It recognizes currency notes of different denomination based on the various unique features extracted. In this method comparison of the values of the features with the predefined set of features is done. If the values fall within the specified threshold range, the denomination will get detected.

\section{TYPES OF CURRENCY RECOGNITION TECHNIQUES}

\subsection{Texture based Recognition Technique}

Texture based currency recognition is a very useful feature. The texture provides the measure of properties such as smoothness, coarseness and regularity. To describe the texture of a region the three principal approaches used in image processing are statistical, structural and spectral. Statistical approaches yield characterizations of textures as smooth, coarse, grainy and so on. Structural techniques deal with description of texture based on regularly spaced lines.

Spectral techniques are based on properties of Fourier spectrum and are used primarily to detect global periodicity in an image [13].Some texture features that have been used for image retrieval are Tamura features, MRSAR, edge histogram, Gabor texture feature, and pyramid-structured and tree-structured wavelet transform. Tamura features define the coarseness, directionality and contrast of an image. Tamura coarseness is the measure of average of coarseness at each pixel location inside the texture region. The performance of this feature is not good since it compute directly from image. So the performance can be improved by using histogram. MRSAR (Multi Resolution
Simultaneous Auto Regressive) gives results which is helpful in distinguishing different texture patterns. Gabor feature extract information at multiples scales and orientation. According to author MRSAR and Gabor feature give better result than other feature. Visual texture doesn't have any strict definition and it is generally the presence of repetitive patterns in which elements or primitives are arranged according to some placement rule [13].

\subsection{Pattern based Recognition Techniques}

Pattern based recognition is basically classification of objects based on some set of images. It requires experimenter to have some prior knowledge about the objects to come to a conclusion. It also requires a large collection of data. There are many techniques that make use of pattern recognition. All these techniques uses vector quantization based histogram modeling. Vector quantization is a sampling method where each point $\mathrm{x}_{\mathrm{j}}$ in a set of data in $\mathrm{d}$-dimensional space is replaced by $\mathrm{L}$ prototype points. The prototype points are picked in such a way that sum of the distances (called the distortion) from each data points $\mathrm{x}_{\mathrm{j}}$ to its nearest prototype point is minimized.

Some work has been done in this area by Seth Mc Neillin for recognition of coin by pattern recognition. In this process first he collects the data by collecting coins with different background. Next the segmentation and cropping is done. In this step coins are segmented from background by using modified Nechba's code. Cropping of the image is done by locating the edges of the coin. Then features were extracted by convolving texture templates with each image. Next step is the training the program with as many data as possible to get high accuracy. Here the author uses five set and get $94 \%$ accuracy [13].

The work in this area is done by Vipin kumar Jain for Indian currency note. In this process he uses digital image processing techniques to find the region of interest and after that Neural Network and pattern recognition techniques is used for matching the pattern. From the experiment he found that recognition method of Indian currency note is quite simple, efficient and easy to be realized because denomination numerals are used for identification which can be extracted easily from paper currency. Then extracted numerals are matched and are found exact match for identification. By using this recognition technique counting of different denomination note in a bunch can be done [14].

Dipti Pawade et al.(2013) made a comparative study on various currency recognition technique. In one of the paper by Kalyan Kumar Debnath et al proposed a method for currency recognition using ensemble neural network(ENN) particularly for Bangladesh currency. Ensemble neural network consist of number of neural network and each neural network is trained independently. Here each neural network is trained via negative correlation learning. The system is able to recognize highly noisy or old image and reduces the misclassification of notes [15]. 
Faiz M. Hasanuzzaman et al. proposed a component based framework for banknote recognition by using Speeded Up Robust Features (SURF). SURF feature divides the image into components and then matches test image with original image component. The SURF features are invariant to conditions of image occlusion, image rotation, changes of scaling, illumination and the viewpoint. The proposed algorithm achieves $100 \%$ accuracy [16].

In paper [17] the author proposed a method of currency recognition using Neural Network. Here they have used color, size and texture as three features that have used to recognize the currency note. In the experimentation they have achieved $97.34 \%$ accuracy.

\subsection{Colour based Recognition Techniques}

Variety of colors is used in each paper currency but there is only one or two dominant color present in each paper currency which can be used for distinguishing each paper currency. The image delivered from the digital camera is in RGB format. The electronic processing of these color signals (Red, Green and Blue) can take a number of different representation formats denoted as color spaces. There are variety of color spaces YIQ, HSV, YUV, HIS and $\mathrm{YCrCb}$ and each format has its own advantage. The choice of color space has a great influence on the performance of procedures such as segmentation. After taking the image from the digital camera the dimensionality of data is reduced. Then the image is converted from the RGB color space to a color space suitable for processing. After processing the features are extracted, matched and currency is detected as genuine or counterfeit.

In 2012 Pragati D. Pawar and Shrikant B. Kale converted image from RGB color space to HSV color space before feature extraction. They used HSV color space because it is close to human conceptual understanding of color. The feature extraction is done by analyzing color histogram, hue, saturation and intensity value. Then color histogram, hue, saturation and intensity value of input image is compared with the saved images. If the difference in threshold value is greater than the specified value then the currency is genuine otherwise counterfeit [18].

Histogram describes the global color distribution in an image by counting the number of pixels of each color. Color histogram method will suit when we have to segregate between the range of color and prominent color. The major limitation of color histogram is that color histogram describes which colors are present in the image and in what quantity but doesn't provide spatial information [10].

John R Smith and Shih Fu Chang has also done work in this area. They propose the techniques for color image retrieval. Here color indexing techniques is used by which images are extracted on the basis of color content of the image. The color indexing algorithm uses back-projection of binary color sets to extract color region from the image. It overcomes some of the problem with color histogram [19]
The major limitation of color based recognition techniques is to recognize an old note whose color become fade or changed due to rigorous circulation.

The three currency recognition techniques discussed above requires a user to always carry a machine along with him her which consumes a lot of power. To overcome the problem a new technique has been developed known as currency localization technique.

\subsection{Currency Localization Techniques}

Currency localization technique is a mixed approach where first currency note in an image is localized and then various threshold based algorithm are applied to determine the denomination of currency note.

In this technique first image is taken by a mobile camera or a webcam. Then some enhancement of the image is required by applying some pre-processing technique such as noise reduction, normalization and contrast enhancement. Next background subtraction, RGB to gray conversion and edge detection is performed. Localization of currency note is done by applying scan line algorithm on the image after edge detection. The image is scanned from left to right and top to bottom line by line. The line is highlighted which contain number of pixels greater than the set threshold. With the intersection of these lines a rectangle is formed which surrounds the currency note. Then color matching technique is used for currency recognition.

Hanish and Padam has done currency recognition in color images by applying currency localization technique. In this experiment they got $96 \%$ efficiency. The system can be enhanced by using template matching [13].

Parminder Sigh Reel, Gopal Krisnan and Smarti Kotwal has done some work on currency note recognition based on heuristic analysis of character and digits of serial number. Heuristic analysis is needed to filter non- character elements which are not reliably separated by traditional OCR method. Heuristic analysis of color separates character and noncharacter elements based on color basis [20].

Suriya Singh et al presented an application for Indian currency recognition on mobile. For this they have used visual Bag of Words (BoW) recognition method. Here they have used GrabCut algorithm to segment the foreground from background. This process of segmentation work so well that it can segment image from the cluttered background. After testing 2584 images they have got $96.7 \%$ accuracy [21].

In [22], the authors proposed a method for currency note recognition by using ANN. Here a special linear transformation function is adapted to wipe out the noise pattern from background without affecting the note original feature. The edge detection feature along with linear transformation is a better feature extraction technique and helps to keep notes look similar in varying conditions. 


\section{CONCLUSION}

Detecting the authenticity and recognition of currency note has become important nowadays because of the prevailing fraudulent activities as it hampers our economy. It is very useful in the banking system as well as in other commercial applications.

There are many methods used to detect authenticity and to recognize a note which is being discussed in this paper and each one has its own objective and significance. In this paper we have discussed many tools that can be used to recognize different features of a note. Since a thing can't be perfect and has certain limitation. So selection of feature and the selection of tools to recognize that particular feature are critical. To overcome this limitation and building an interactive system with high speed, accuracy and cheap is a new challenge.

\section{REFERENCES}

[1]. Sanjana, Manoj Diwakar and Anand Sharma, "An Automated recognition of fake or destroyed Indian currency note in machine vision", International Journal of Computer Science and Management Studies, ISSN 2231-5268, Vol 12, Issue 2, April 2012.

[2]. Reserve Bank Of India, Chennai, "Essential and security features of Banknotes"

[3]. D. Alekhya, G. Devi Surya Prabha and G. Venkata Durga Rao,"Fake currency detection using image processing and other standards methods", ISSN 2278- 5841, Vol 3, Issue 1, Jan 2014.

[4]. Kishan Chakroborty, Jordan Bsumatary, Debasmita Dasgupta, Jagadish Chandra Kalita and Subra Mukherjee, "Recent Developments in Paper currency Recognition", e ISSN 2319-1163, p ISSN 2321-7308, Vol 2, Issue 11, Nov 2013.

[5]. Rubeena Mirza and Vinti Nanda, "Paper currency Verification system based on Characteristics Extraction using image processing", International Journal of Engineering and Advance Technology, ISSN 2249-8958, Vol 1, Issue 3, Feb 2012.

[6]. Rubeena Mirza and Vinti Nanda, "Design and implementation of Indian paper currency Authentication system based on feature extraction by edge based segmentation using Sobel operator", International Journal of Engineering Research 7 Development e-ISSN 2278-076X, p-ISSN 2278-800X, Vol 3, issue 2.

[7]. Ankush Roy, Biswajit Halder and Utpal Garain, "Authentication of Currency Notes through Printing Technique Verification”, ICVGIP'10, Dec 12-05-2010.

[8]. Ankush Roy, Biswajit Halder, Utpal Garain and David S Doermann, "Machine Assisted Authentication of Paper Currency: an Experiment on Indian Banknotes ", cs.CV Jan 2014.

[9]. Arcangelo Bruna, Giovanni Maria Farinella, Giuseppe Claudio Guarnera and Sebastiano Battiato, "Forgery Detection and Value Identification of Euro Banknotes", ISSN 1424-8220, Sensors 2013, 13, 2515-2529.
[10]. Archana Bade, Deepali Aher and Prof Smita Kulkarni, " Note to coin exchanger using image processing", International Journal of Recent and Innovation Trends in Computing \& Communication, ISSN 2321-8169, Vol 1, Issue 3.

[11]. A. Ms. Trupti Pathrabe and B. Dr. N. G. Bawane," Paper Currency Recognition System using Characteristics Extraction and Negatively Correlated NN Ensemble ", International Journal of Latest Trends in Computing, EISSN: 2045-5364, Volume 1, Issue 2, December 2010.

[12]. Ahmed Ali and Mirfa Manzoor, "Recognition System for Pakistani Paper Currency", Research Journal of Applied Sciences, Engineering and Technology 6, ISSN 2040-7459; e-ISSN 2040-7467, Sep 2013.

[13]. Hanish Agarwal and Padam Kumar,"Indian currency note Denomination recognition using color images", International Journal of Computer Engineering and Communication Technology, ISSN 2278-5140, Vol 1, Issue 1.

[14]. Vipin Kumar Jain and Dr Ritu Vijay, "Indian Currency Denomination Identification Using Image Processing Technique", International Journal of Computer Science and Information Technologies, ISSN 0975-9646, Vol 4, Issue 1, 2013.

[15]. Dipti Pawade, Pranchal Chaudhari and Harshada Sonkamble, "Comparitive Study of Different Paper currency and coin currency Recognition Method", International Journal of Computer Application, ISSN 0975-8887, Vol 66, No 23, Mar 2013.

[16]. Faiz M. Hasanuzzaman, Xiaodong Yang, and YingLi Tian, "Robust and Effective Component-based Banknote Recognition by SURF Features".

[17]. Rumi Ghosh and Rakesh Khare," An Elegant Neural Network based draw near for currency Recognition", Journal of Environmental Science, Computer Science and Engineering and Technology, JECET; June - August-2013; Vol.2.No.3, 876-882.

[18]. Pragati D Pawar and Shrikant B. Kale," Recognition of Indian Currency Note Based on HSV Parameters", ISSN 2319-7064, Vol 3, Issue 6, Jun 2014.

[19]. John R. Smith and Shih-Fu Chang, "Tools and Techniques for Color Image Retrieval", IS\&T/SPIE Proceedings Vol. 2670.

[20]. Parminder Singh Reel, Gopal Krishnan and Smarti Kotwal, "Image processing based heuristic analysis for enhanced currency recognition", International Journal of Advancements in Technology, ISSN 0976-4860, Vol 2, Issue 1, Jan 2011.

[21]. Suriya Singh, Shushman Choudhury, Kumar Vishal and C.V. Jawahar, "Currency Recognition on Mobile Phones".

[22]. D.K.A.S.Gunaratna, N.D.Kodikara and H.L.Premaratne," ANN Based Currency Recognition System using Compressed Gray Scale and Application for Sri Lankan Currency Notes-SLCRec", International Journal of Computer, Information, Systems and Control Engineering Vol:2 No:9, 2008. 